\title{
Hormonal Responses to Cold Exposure in Subjects with Vibration Syndrome
}

\author{
NORIAKI HARADA, MINORU NAKAMOTO, HIDEAKI KOHNO, \\ HIROKAZU KONDO AND MORISHIGE TANAKA* \\ Departments of Public Health and Central Research Laboratory*, Ehime \\ University School of Medicine, Shigenobu, Ehime, 791-02 Japan
}

Received for publication May 22, 1989

\begin{abstract}
Summary: Responses of the autonomic nervous system and the endocrine system to cold exposure were investigated. The subjects were all males, between 41 and 69 years of age. Seventy subjects with the vibration syndrome and vibration-induced white finger $(\operatorname{VWF}(+)$ group), 70 subjects with the vibration syndrome without $\operatorname{VWF}(\operatorname{VWF}(-)$ group) and 70 healthy subjects (control group) were individually matched according to age. The subjects remained in a cold room at $7^{\circ} \mathrm{C}$ for 30 minutes. Catecholamines, cyclic nucleotides, thyroid hormones, ACTH and cortisol were measured before and during cold exposure. The $\operatorname{VWF}(+)$ group and the $\operatorname{VWF}(-)$ group had larger $\%$ increases of plasma norepinephrine, cyclic AMP, cyclic GMP, $\mathrm{T}_{3}$ and $\mathrm{T}_{4}$ levels than the control group. These findings indicate that the responses of the autonomic nervous system and the thyroid system to cold exposure are greater in the subjects with the vibration syndrome.
\end{abstract}

Key words: plasma level-catecholamines-cyclic nucleotides-thyroid hormones-ACTH-cortisol-vibration-induced white finger

\section{Introduction}

The effects of occupational hand-arm vibration on the regulatory systems of the body were investigated by monitoring autonomic nervous function and endocrine function using electrophysiological methods and blood chemical analyses during exposure to cold. This included heart rate variation obtained from an electrocardiogram (Harada et al. 1987) and a power spectrum of the microvibration on thenar eminence (Harada and Kondo, 1986). Dopamine-beta-hydroxylase activity (Harada et al. 1989), cyclic nucleotids (Harada et al. 1985), catecholamines, pituitary hormones, thyroid hormones and cortisol (Kondo, 1988; Harada et al. 1988) were measured in the blood. The results indicated that the basal activity of the regulatory systems and the responses to cold exposure were different for subjects with vibration syndrome and control subjects.

A further investigation was performed using not only these methods, but also indices of the central nervous system on a large number of subjects with agematched controls. The data are still being analyzed. In this paper, the changes of hormonal substances in the blood, such as catecholamines (norepinephrine, epinephrine), plasma cyclic nucleotides (cyclic adenosine 3' 5'-monophosphate, cyclic AMP; and cyclic guanosine 3' 5'-monophosphate, cyclic GMP), thyroid hormones (triiodothyronine, $\mathrm{T}_{3}$; thyroxine, $\mathrm{T}_{4}$ ), adrenocorticotropic hormone (ACTH) and 
cortisol, during exposure to cold are described.

\section{Subjects and Methods}

Eighty-six subjects with the vibration syndrome and vibration-induced white finger (VWF, VWF (+) group), 97 subjects with the vibration syndrome without VWF (VWF (-) group) and 84 healthy subjects (control group) were examined in four areas of western Japan. All subjects were males, between 41 and 69 years of age, who did not have pneumoconiosis, diabetes mellitus, hypertension, heart disease, liver disease nor a history of cerebrovascular disease. They were individually matched according to age, within a 5 year range, among the three groups. Also, when comparing the VWF $(+)$ and VWF (-) groups, the subjects were individually matched within a 3 year range according to the number of years which had elapsed since ceasing the operation of vibratory tools.

As a result of the selection process, 70 subjects with the vibration syndrome and VWF, 70 subjects with the vibration syndrome without VWF and 70 healthy subjects were obtained. Means of age, body height, body weight, years of exposure to vibration and years after ceasing the operation of vibratory tools were not significantly different for the three groups (Table 1). The most common tools which had been operated by the subjects of the VWF (+) and VWF (-) groups were chain-saws (61 workers, 44\%) and rock drills (42 workers, $30 \%$ ). The number of matched sets which were actually analyzed were different for each hormonal substance because it was not possible to measure catecholamines and $\mathrm{T}_{4}$ in some samples, and measurements of ACTH and cortisol were limited to one of the four geographic areas.

Blood samples were taken under two conditions. At first, the subject was required to sit in a room at a temperature of $25 \pm 1^{\circ} \mathrm{C}$ and blood was drawn after 25 minutes. Then the subject moved and sat down in a cold room in which the temperature was controlled at $7 \pm 1.5^{\circ} \mathrm{C}$ and blood was drawn after 25 minutes (cold exposure test). For this test, the subjects wore two pieces of clothing on the upper and lower half of the body. The plasma was separated from the blood using a centrifugal separator at $4^{\circ} \mathrm{C}$ and was frozen at $-70^{\circ} \mathrm{C}$. Catecholamines were analyzed using HPLC with the THI method. Other hormones were analyzed using an RI assay kit (Yamasa for cyclic nucleotides, Dainabot for $\mathrm{T}_{3}$, cortisol and $\mathrm{T}_{4}$ and CIS for ACTH). Drawing of blood was performed from 9:30 am to $12: 30$ am from October, 1987 to March, 1988. For 7 days before the tests, all drug treatments were terminated. For the statistical test, the Wilcoxon signed-rank test was used and the significance levels were 0.05 and 0.01 .

TABLE 1

Number of subjects, ages, body dimensions, periods of exposure to vibration and periods since ceasing the operation of vibratory tools.

\begin{tabular}{lcccccc}
\hline & $\mathrm{n}$ & $\begin{array}{c}\text { age } \\
(\mathrm{yr})\end{array}$ & $\begin{array}{c}\text { height } \\
(\mathrm{cm})\end{array}$ & $\begin{array}{c}\text { weight } \\
(\mathrm{kg})\end{array}$ & $\begin{array}{l}\text { years of } \\
\text { exposure }\end{array}$ & $\begin{array}{l}\text { years after } \\
\text { ceasing }^{\mathrm{A}}\end{array}$ \\
\hline VWF $(+)$ group & 70 & $55.9 \pm 5.6$ & $161.7 \pm 5.4$ & $58.6 \pm 7.3$ & $21.2 \pm 7.8$ & $5.6 \pm 3.5$ \\
VWF $(-)$ group & 70 & $55.9 \pm 5.9$ & $160.4 \pm 6.3$ & $59.7 \pm 8.4$ & $19.8 \pm 7.5$ & $5.5 \pm 3.6$ \\
coutrol group & 70 & $55.7 \pm 6.1$ & $161.0 \pm 6.7$ & $60.8 \pm 9.1$ & & \\
\hline
\end{tabular}

Mean $\_\mathrm{SD}$; $A$ excluding 37 subjects in each group who are still operating tools. 


\section{Results}

Table 2 shows the changes of plasma norepinephrine levels during cold exposure. The plasma norepinephrine increased during cold exposure in all three groups $(p<0.01)$. The $\%$ increase for the $\operatorname{VWF}(+)$ group was the largest and the increase for the VWF (-) group was next. The \% increases of the VWF $(+)$ and VWF $(-)$ groups were significantly different from the control group $(p<0.01, p<0.05)$. Table
3 shows the changes of plasma epinephrine during cold exposure. The plasma epinephrine in the VWF $(-)$ and control groups decreased during cold exposure $(\mathrm{p}<0.01)$, while the level in the VWF $(+)$ group did not change significantly.

Table 4 shows the changes of plasma cyclic AMP concentrations during cold exposure. The increase in cyclic AMP induced by cold exposure was statistically significant in all three groups $(\mathrm{p}<0.01)$, however the \% increases in the $\mathrm{VWF}(+)$

TABLE 2

Responses of plasma norepinephrine levels $(\mathrm{pg} / \mathrm{ml}$ ) to a cold exposure test of the whole body at $7^{\circ} \mathrm{C}$.

\begin{tabular}{lcccc}
\hline & $\mathrm{n}$ & rest & exposure & $\%$ increase \\
\hline VWF $(+)$ group & 65 & $326 \pm 133$ & $1027 \pm 384 \# \#$ & $234 \pm 119^{* *}$ \\
VWF $(-)$ group & 65 & $320 \pm 120$ & $951 \pm 391 \# \#$ & $205 \pm 91^{*}$ \\
control group & 65 & $352 \pm 121$ & $926 \pm 296 \# \#$ & $173 \pm 72$ \\
\hline
\end{tabular}

Mean $\pm \mathrm{SD}$; \#\# $\mathrm{p}<0.01$ comparison between rest and exposure.

$* \mathrm{p}<0.05, \quad * * \mathrm{p}<0.01$ compared with the control group.

TABLE 3

Responses of plasma epinephrine levels $(p g / m l)$ to a cold exposure test of the whole body at $7^{\circ} \mathrm{C}$.

\begin{tabular}{lcccr}
\hline & $\mathrm{n}$ & rest & exposure & \% increase \\
\hline VWF $(+)$ group & 65 & $91.3 \pm 53.2$ & $81.0 \pm 47.7$ & $3.2 \pm 58.2$ \\
VWF $(-)$ group & 65 & $87.4 \pm 49.0$ & $73.9 \pm 38.0 \# \#$ & $-8.5 \pm 34.5$ \\
control group & 65 & $96.2 \pm 70.5$ & $72.2 \pm 42.0 \# \#$ & $-13.4 \pm 33.6$ \\
\hline
\end{tabular}

Mean \pm SD; \#\# $\mathrm{p}<0.01$ comparison between rest and exposure.

TABLE 4

Responses of plasma cyclic AMP levels ( $p m o l / m l$ ) to a cold exposure test of the whole body at $7^{\circ} \mathrm{C}$.

\begin{tabular}{lcccc}
\hline & $\mathrm{n}$ & rest & exposure & $\%$ increase \\
\hline VWF $(+)$ group & 70 & $16.3 \pm 4.0$ & $19.1 \pm 5.4 \# \#$ & $17.7 \pm 16.5^{*}$ \\
VWF $(-)$ group & 70 & $15.4 \pm 3.9^{* *}$ & $18.3 \pm 5.0 \# \#$ & $19.0 \pm 17.6^{*}$ \\
control group & 70 & $16.6 \pm 4.3$ & $18.7 \pm 5.1 \# \#$ & $12.9 \pm 17.5$ \\
\hline
\end{tabular}

Mean $\pm \mathrm{SD}$; \#\# $\mathrm{p}<0.01$ comparison between rest and exposure.

$* \mathrm{p}<0.05, \quad * * \mathrm{p}<0.01$ compared with the control group. 
and VWF (-) groups were larger than that of the control group $(\mathrm{p}<0.05)$. Table 5 shows the changes of cyclic GMP during cold exposure. The increase of cyclic GMP induced by cold exposure was statistically significant in all three groups $(p<0.01)$. The $\%$ increases in the VWF $(+)$ and VWF (-) groups tended to be larger than that of the control group, however the differences were not statistically significant.

Table 6 shows the changes of plasma
$\mathrm{T}_{3}$ during cold exposure. The plasma $\mathrm{T}_{3}$ concentrations for the VWF $(+)$ and VWF (-) groups at rest tended to be smaller than for the control group. Cold exposure induced an increase of plasma $\mathrm{T}_{3}$ in all three groups $(\mathrm{p}<0.01)$. The $\%$ increases for the VWF $(+)$ and VWF $(-)$ groups were larger than the increases in the control group $(p<0.05)$. Table 7 shows the changes of plasma $\mathrm{T}_{4}$ during cold exposure. The plasma $\mathrm{T}_{4}$ concentrations in the VWF $(+)$ and VWF $(-)$ groups at

TABLE 5

Responses of plasma cyclic GMP levels ( $p m o l / m l$ ) to a cold exposure test of the whole body at $7^{\circ} \mathrm{C}$.

\begin{tabular}{lcccc}
\hline & $\mathrm{n}$ & rest & exposure & \% increase \\
\hline VWF $(+)$ group & 70 & $3.02 \pm 1.39$ & $3.70 \pm 1.68 \# \#$ & $35.2 \pm 81.2$ \\
VWF $(-)$ group & 70 & $2.96 \pm 1.47$ & $3.75 \pm 2.48 \# \#$ & $34.3 \pm 84.4$ \\
control group & 70 & $2.95 \pm 1.52$ & $3.41 \pm 1.59 \# \#$ & $24.9 \pm 43.7$ \\
\hline
\end{tabular}

Mean $\pm \mathrm{SD}$; \#\# $\mathrm{p}<0.01$ comparison between rest and exposure.

TABLE 6

Responses of plasma $T_{3}$ levels $(\mathrm{ng} / \mathrm{ml})$ to a cold exposure test of the whole body at $7^{\circ} \mathrm{C}$.

\begin{tabular}{lcccc}
\hline & $\mathrm{n}$ & rest & exposure & $\%$ increase \\
\hline VWF $(+)$ group & 70 & $1.14 \pm 0.55$ & $1.29 \pm 0.60 \# \#$ & $17.6 \pm 19.5^{*}$ \\
VWF $(-)$ group & 70 & $1.15 \pm 0.53$ & $1.30 \pm 0.55 \# \#$ & $16.6 \pm 22.0^{*}$ \\
control group & 70 & $1.24 \pm 0.53$ & $1.34 \pm 0.59 \# \#$ & $10.2 \pm 20.1$ \\
\hline
\end{tabular}

Mean \pm SD; \#\# $\mathrm{p}<0.01$ comparison between rest and exposure.

$* \mathrm{p}<0.05$ compared with the control group.

TABLE 7

Responses of plasma $T_{4}$ levels $(\mu \mathrm{g} / 100 \mathrm{ml})$ to a cold exposure test of the whole body at $7^{\circ} \mathrm{C}$.

\begin{tabular}{lcccl}
\hline & $\mathrm{n}$ & rest & exposure & \% increase \\
\hline VWF $(+)$ group & 69 & $9.91 \pm 4.72$ & $11.44 \pm 5.97 \# \#$ & $14.0 \pm 17.3$ \\
VWF $(-)$ group & 69 & $9.46 \pm 4.60^{*}$ & $11.22 \pm 5.58 \# \#$ & $19.6 \pm 22.5^{* *}$ \\
control group & 69 & $10.94 \pm 4.67$ & $12.16 \pm 6.23 \# \#$ & $10.7 \pm 18.5$ \\
\hline
\end{tabular}

Mean $\pm \mathrm{SD}$; \#\# $\mathrm{p}<0.01$ comparison between rest and exposure.

$* \mathrm{p}<0.05, * * \mathrm{p}<0.01$ compared with the control group. 
rest and during cold exposure tended to be smaller than those in the control group. An increase of plasma $\mathrm{T}_{4}$ induced by cold exposure was observed in all three groups $(\mathrm{p}<0.01)$. The $\%$ increases for the $\operatorname{VWF}(+)$ and VWF (-) groups tended to be larger than those in the control group. The difference between the VWF (-) group and the control group was statistically significant $(\mathrm{p}<0.01)$.

Table 8 shows the changes of plasma ACTH during cold exposure. The plasma $\mathrm{ACTH}$ of the VWF (+) group was the lowest and that of the VWF (-) group was intermediate for each condition. The difference of plasma ACTH during rest for the VWF $(+)$ group and the control group was statistically significant $(\mathrm{p}<0.05)$. Cold exposure induced a decrease of the plasma ACTH in all three groups, however only the decrease in the control group was statistically significant $(p<0.05)$. The change of plasma ACTH in the VWF $(+)$ group was the smallest and that in the VWF (-) group was intermediate; however the differences between these changes and the change for the control group were not statistically significant. Table 9 shows the changes of plasma cortisol during cold exposure. The concentrations of plasma cortisol for the three groups at rest were almost the same. The absolute plasma cortisol concentration and the $\%$ increase during cold exposure tended to be larger in the VWF (-) group than in the other two groups; however the differences were not statistically significant.

TABLE 8

Responses of plasma ACTH levels $(\mathrm{pg} / \mathrm{ml})$ to a cold exposure test of the whole body at $7^{\circ} \mathrm{C}$.

\begin{tabular}{ccccc}
\hline & $\mathrm{n}$ & rest & exposure & $\%$ increase \\
\hline VWF $(+)$ group & 12 & $26.3 \pm 20.1^{*}$ & $23.6 \pm 19.8$ & $-6.6 \pm 42.0$ \\
VWF $(-)$ group & 12 & $34.3 \pm 26.6$ & $29.0 \pm 20.7$ & $-9.9 \pm 62.7$ \\
control group & 12 & $43.2 \pm 19.8$ & $33.3 \pm 15.7 \#$ & $-19.9 \pm 28.1$ \\
\hline
\end{tabular}

Mean $\pm \mathrm{SD} ; \# \mathrm{p}<0.05$ comparison between rest and exposure.

* $\mathrm{p}<0.05$ compared with the control group.

TABLE 9

Responses of plasma cortisol levels ( $\mathrm{pg} / \mathrm{ml}$ ) to a cold exposure test of the whole body at $7^{\circ} \mathrm{C}$.

\begin{tabular}{lcccr}
\hline & $\mathrm{n}$ & rest & exposure & $\%$ increase \\
\hline VWF $(+)$ group & 12 & $125.2 \pm 39.8$ & $119.9 \pm 35.6$ & $0.0 \pm 32.1$ \\
VWF $(-)$ group & 12 & $127.8 \pm 48.9$ & $132.2 \pm 40.9$ & $11.8 \pm 48.7$ \\
control group & 12 & $129.3 \pm 51.4$ & $123.4 \pm 50.2$ & $-3.4 \pm 19.4$ \\
\hline
\end{tabular}




\section{Discussion}

To evaluate plasma levels of hormones, the circadian rhythm must be considered. This is especially important for plasma cortisol levels (Weitzman et al. 1975). In this study, blood samples were taken during a three hour interval before noon, and differences of plasma levels before and during cold exposure were evaluated. Therefore, the effects of the circadian rhythm on the results should be small.

Table 10 summarizes the hormonal responses to cold exposure in subjects with vibration syndrome and in healthy controls. Evaluation of the responses is based on the statistical significance of the differences of the results during rest and during cold exposure and comparison of the $\%$ increases with the control group. Cold exposure induced increases of plasma norepinephrine, cyclic AMP, cyclic GMP, $\mathrm{T}_{3}$ and $\mathrm{T}_{4}$, and decreases of plasma epinephrine and ACTH in the control group. The increases were usually larger in the VWF (+) and VWF (-) groups and a decrease for these two groups was only observed for the plasma epinephrine level of the VWF (-) group.

Plasma norepinephrine is maintained by release from the sympathetic nerve endings and is a sensitive indicator of sympathetic nerve function. The results with plasma norepinephrine indicate that the sympathetic nervous system in subjects with the vibration syndrome is easily activated by cold exposure. It is interesting that norepinephrine has a strong action to contract the smooth muscle of vessels and that VWF is provoked when the whole body is exposed to cold.

The plasma epinephrine levels in the VWF (-) and control groups decreased during cold exposure. Epinephrine is secreted primarily by the adrenal medulla and the control of release is not the same as the control for norepinephrine release. The discrepancy between the responses of plasma norepinephrine and epinephrine to cold exposure can be explained by this "selective control" (Brück, 1983). The fact that a significant decrease of plasma epinephrine was not observed in the VWF $(+)$ group may be related to a hypersensitivity of the VWF $(+)$ subjects to cold.

Cyclic AMP and cyclic GMP are second messengers in cells, and changes in blood concentrations during physiological stress are pointed out to reflect sympathetic beta receptor function and sympathetic alpha or parasympathetic muscarinic receptor

TABLE 10

Summary of hormonal responses to cold exposure in subjects with the vibration syndrome and control subjects.

\begin{tabular}{|c|c|c|c|c|c|c|c|c|}
\hline & \multicolumn{2}{|c|}{ catecholamines } & \multicolumn{2}{|c|}{ cyclic nucleotides } & \multicolumn{2}{|c|}{ thyroid hormones } & \multirow{2}{*}{ ACTH } & \multirow{2}{*}{ cortisol } \\
\hline & $\mathrm{NE}$ & $\mathrm{EP}$ & cAMP & cGMP & $\mathrm{T}_{3}$ & $\mathrm{~T}_{4}$ & & \\
\hline $\mathrm{n}$ of each group & 65 & 65 & 70 & 70 & 70 & 69 & 12 & 12 \\
\hline VWF $(+)$ group & $\uparrow \uparrow \uparrow$ & - & $\uparrow \uparrow$ & $\uparrow$ & $\uparrow \uparrow$ & $\uparrow$ & - & - \\
\hline VWF (-) group & $\uparrow \uparrow$ & $\downarrow$ & $\uparrow \uparrow$ & $\uparrow$ & $\uparrow \uparrow$ & $\uparrow \uparrow$ & - & - \\
\hline control group & $\uparrow$ & $\downarrow$ & $\uparrow$ & $\uparrow$ & $\uparrow$ & $\uparrow$ & $\downarrow$ & - \\
\hline
\end{tabular}

$\uparrow$ increase; $\downarrow$ decrease; - no change.

NE norepinephrine; EP epinephrine; cAMP cyclic AMP; cGMP cyclic GMP.

Evaluations were based on the statistical significance of each difference between rest and exposure conditions, and the comparison of the $\%$ increase with the control group. 
functions, respectively (Okada et al. 1980; Ui et al. 1980). It has been reported that an increase in plasma cyclic GMP was induced by immersing a hand in cold water in vibration syndrome patients but not in healthy controls (Okada et al. 1983). The changes of plasma cyclic AMP and cyclic GMP levels indicate that the response of receptors on nerve endings to cold exposure in subjects with the vibration syndrome are larger. These phenomena probably relate to a hyperfunction of the autonomic nervous system.

Cold exposure induces an enhancement of $\mathrm{T}_{3}$ - and $\mathrm{T}_{4}$ - production in laboratory animals. It was pointed out that similar responses of plasma $T_{3}$ and $T_{4}$ to acute cold exposure had not been confirmed in man (Sterling and Lazarus, 1977). However, a recent study showed that cold exposure of healthy subjects to $4^{\circ} \mathrm{C}$ for 30 minutes induced a rise of circulating $\mathrm{T}_{3}$ and $\mathrm{T}_{4}$ levels. This rise was interpreted to be the result of a direct effect of increased norepinephrine on the thyroid gland (O’Malley BP et al. 1984). These findings coincide with the present results. Significant increases of plasma $\mathrm{T}_{3}$ and $\mathrm{T}_{4}$ levels were induced by cold exposure in all three groups. The $\%$ increases in the VWF (+) and VWF (-) groups were larger than for the control group. These results indicate that the sensitivity to cold of subjects with the vibration syndrome is higher than the sensitivity of control subjects.

The plasma ACTH levels tended to decrease during cold exposure and the plasma cortisol levels did not change significantly in any of the groups. Many acute stresses can increase the secretion of ACTH from the pituitary, which then activates the adrenal cortex. The following reasons may account for the fact that positive results were not observed. First, the number of subjects in which plasma $\mathrm{ACTH}$ and cortisol levels were measured was small. Second, the severity of cold stress in this study may not have been sufficient to induce an "alarm response" in the pituitary-adrenal cortical system.

\section{Conclusion}

The VWF (+) and VWF (-) groups had larger $\%$ increases of plasma norepinephrine, cyclic AMP, cyclic GMP, $\mathrm{T}_{3}$ and $\mathrm{T}_{4}$ levels than the control group. These findings indicate that the responses of the autonomic nervous system and the thyroid system to cold exposure are enhanced in subjects with the vibration syndrome.

This work was supported by a grant-in-aid for scientific research from the Ministry of Education, Science and Culture of Japan.

\section{References}

BRUCK, K. (1983). Functions of the endocrine system. In Human Physiology. pp 658-687. Berlin: Springer-Verlag.

Harada, N. and Kondo, H. (1986). Microvibration on thenar eminence and autonomic nervous function in patients with vibration syndrome. The Autonomic Nervous System 23, 490-495. (in Jpn with Eng abstract).

Harada, N., Kondo, H. and Kimura, K. (1985). Autonomic nervous function in patients with vibration syndrome-Plasma cyclic nucleotide responses to cold-exposure test. Igakunoayumi 135, 659-670. (in Jpn).

Harada, N., Kondo, H. and Kimura, K. (1987). Autonomic nervous function in patients with vibration syndrome-An investigation of $R-R$ interval on ECG and microvibration on thenar eminence. Jpn. J. Ind. Health 29, 152-153. (in Jpn).

Harada, N., Kondo, H. and Kimura, K. (1988). Pituitary-thyroid function in patients with vibration syndrome. Jpn. J. Ind. Health 30, 136-137. (in Jpn).

Harada, N., Yoshida, I. and Kimura, K. (1989). Heart rate variation and serum dopaminebeta-hydroxylase activity in workers exposed 
to vibration. Int. Arch. Occup. Environ. Health 61, 369-373.

Kondo, H. (1988). Functions of the sympatheticadrenomedullar system and adrenocortex in patients with vibration syndrome. Jpn. J. Ind. Health 30, 263-271. (in Jpn with Eng abstract).

OKadA, F., Honma, M. and Ui, M. (1980). Changes in plasma cyclic nucleotides levels during various acute physical stresses. Horm. Metab. Res. 12, 80-83.

OKadA, F., KiYota, N., Honma, M. and Ui, M. (1983). Plasma guanosine 3', 5' -monophosphate responses to the cold pressor test in patients with vibration disease. Arch. Environ. Health. 38, 144-147.

O’Malley, B. P., Cook, N., Richardson, A., Barnet, D. B. and Rosenthal, F.D. (1984). Circulating catecholamine, thyrotrophin, thyroid hormone and prolactin responses of normal subjects to acute cold exposure. Clin. Endocrinol. 21, 285-291.

Sterling, K. and Lazarus, J.H. (1977). The thyroid and its control. Ann. Rev. Physiol. 39, 349-371.

Ui, M., Honma, M., Kunitada, S., Okada, F., Ide, H., Hata, S. and Satoh, T. (1980). Adrenergic and cholinergic modulation of extracellular cyclic nucleotides. Adv. Cyclic. Nucleotide Res. 12, 25-35.

Weitzman, E.D., Boyar, R. M., Kapen, S. and Hellmann, L. (1975). The relationship of sleep and sleep stages to neuroendocrine secretion and biological rhythms in man. Recent Prog. Horm. Res. 31, 399-446. 